\title{
EL TURISMO DE BASE COMUNITARIA Y LOS PROCESOS DE GOBERNANZA EN LA COMUNA DE PANGUIPULLI, SUR DE CHILE
}

\author{
Mg. Guillermo Pacheco Habert \\ Investigador CEAM \\ Académico Instituto de Turismo \\ Universidad Austral de Chile (UACh) \\ Dr. @ Christian Henríquez Zúñiga \\ Investigador CEAM \\ Académico Instituto de Economía \\ Universidad Austral de Chile (UACh)
}

\section{RESUMEN}

El turismo de base comunitaria (TBC) es una herramienta para que las comunidades logren defender sus territorios de amenazas como por ejemplo, la especulación inmobiliaria y la descaracterización cultural. Con este turismo ha sido posible, la generación de trabajo y renta, proteger la biodiversidad y la identidad cultural, conservando modos de vida de comunidades rurales e indígenas. En Latinoamérica, territorio propicio para este turismo, su aparición ha sido resultado de procesos influenciados por la autogestión y autonomía comunitaria, para hacer frente a las distintas problemáticas que afectan la calidad de vida de las familias y comunidades locales residentes. La hipótesis planteada muestra que el potencial del Turismo de Base Comunitaria puede ser usado como un camino para impulsar procesos de gobernanza local que permitan a las comunidades participar, activamente, en la toma de decisiones junto a los actores públicos y privados que intervienen los territorios turísticos. El objetivo de este artículo es presentar un marco teórico y metodológico a partir de la experiencia de gobernanza llevada a cabo en el contexto del Programa de turismo de base comunitaria, economía solidaria y desarrollo de base local en la comuna de Panguipulli, sur de Chile.

Palabras clave: Turismo de Base Comunitaria, Gobernanza, Territorio. 


\title{
COMMUNITY BASED TOURISM AND GOVERNANCE PROCESS IN PANGUIPULLI COMMUNE, SOUTH OF CHILE
}

\author{
Mg. Guillermo Pacheco Habert \\ Investigador CEAM \\ Académico Instituto de Turismo \\ Universidad Austral de Chile (UACh) \\ Dr. (C) Christian Henríquez Zúñiga \\ Investigador CEAM \\ Académico Instituto de Economía \\ Universidad Austral de Chile (UACh)
}

\begin{abstract}
Community Based Tourism (CBT) is a tool for local residents to defend their territories from threats such as property speculation and cultural decharacterization. With this kind of tourism has been able to protect biodiversity and cultural identity, generate jobs and incomes for families, keeping living ways of rural and indigeneous communities. In Latin America, its appearance has been the result of processes influenced by self-management and community autonomy. The hypothesis raised shows that the potential of community-based tourism can be used as a way to promote processes of local governance that allow to communities to participate in decision-making with the public and private actors involved in the territories. This research aims to present a theoretical and methodological framework from governance experience in context of Programme of Community Based Tourism, Solidarity Economy and Local Development in Panguipulli commune, south of Chile.
\end{abstract}

Key words: Community Based Tourism, Governance, Territory. 


\section{I- Introducción}

En la comuna de Panguipulli, localizada en la Región de Los Ríos, sur de Chile, se desarrolla desde el 2013 el Programa de Turismo de Base Comunitaria, Economía Solidaria y Desarrollo de Base Local, ejecutado por el Centro Transdisciplinario de Estudios Ambientales y Desarrollo Humano Sostenible de la Universidad Austral de Chile (CEAM - UACh). El Programa se ejecuta mediante tres objetivos específicos: 1- Evaluar la inserción del TBC en la región de los Ríos y el sur de Chile, desde un enfoque interdisciplinario e intercultural. 2. Impulsar la cooperación, solidaridad, la autogestión y autodependencia en los territorios y entre las iniciativas. 3. Apoyar mediante asesorías, transferencia tecnológica multidimensional y difusión, a las iniciativas de turismo desarrolladas por residentes y comunidades locales.

A través de este Programa se realiza un trabajo de investigación acción participante con las comunidades locales, especialmente con iniciativas de economía solidaria y turismo de base comunitaria que recientemente vienen incorporándose a la oferta turística de la comuna. En ese sentido, el Programa apoya a organizaciones sociales y de turismo, ferias locales, cocinerías, guías locales, campings, iniciativas de artesanías, huertas locales y vida de campo, vivencias interculturales entre otras. El Programa busca potenciar además la gobernanza en virtud de la conservación de la biodiversidad en base a actividades productivas inspiradas en el buen vivir.

\section{II- Objetivos}

Objetivo General: Describir y complejizar el proceso de gobernanza del turismo de base comunitaria en la comuna de Panguipulli, en un contexto teórico y práctico.

\section{Objetivos específicos:}

- Describir las relaciones entre el paradigma de gobernanza y el turismo de base comunitaria. -Complejizar las problemáticas del TBC que inciden directamente en los procesos sociales que viven organizaciones y comunidades que buscan su participación activa en los sistemas de gobernanza. 


\section{III- El turismo de base comunitaria: Un camino para la gobernanza}

Se trata de un cambio de paradigma de la clásica planificación del turismo, tras comprender que los visitantes no son los únicos sujetos que deben beneficiarse de la actividad. Generalmente la planificación y gestión de destinos es ejecutada bajo alianzas públicas y privadas que en algunas comunas turísticas de Chile ni siquiera funcionan en la práctica. Tampoco ha sido posible incluir a la comunidad local en las gobernanzas del turismo en el sur de Chile (Pacheco et. al 2014). Para la OMT, el turismo se centra principalmente en los viajeros, en su glosario oficial lo define como "el turismo se refiere a una actividad realizada por los visitantes" (OMT 2015), aquí quedan olvidados específicamente quienes reciben, es decir las comunidades locales. Últimamente la definición de turismo según la OMT, ha evolucionado de la visión que vincula al turismo con viajeros con intereses de ocio (turistas), actualmente se relaciona al turismo con las cuentas satélite asociadas a los bienes y servicios demandados por los visitantes en un destino o territorio. Se sigue centrando sólo en el visitante, pero ahora no solo a quienes viajan por motivos de recreación en su tiempo libre, sino principalmente a personas que viajan y demandan bienes y servicios, centrándose en el gasto y en la capacidad de generar dinamización económica.

Diferente de lo anterior, el turismo de base comunitaria se entiende como un fenómeno humano, transdisciplinario e intercultural relativo a los viajes, que se basa de una dialéctica entre anfitriones y visitantes. Según Sampaio (2005) es una estrategia de comunicación social para que comunidades conserven sus modos de vida y junto con ello se conserve la diversidad biocultural. Se compone de elementos fundamentales como la comunidad, cotidianidad y convivencialidad (Henríquez 2010; Skewes et. al. 2012). Y mas allá que esto, permite la defensa del territorio para el buen vivir de las comunidades locales. Es una actividad que es útil como alternativa de renta familiar, sin embargo la renta no es un fin en sí mismo, sino es utilizada como una herramienta comunicacional y socioeconómica para visibilizar y enfrentar amenazas territoriales como los conflictos socioambientales, especulación inmobiliaria ${ }^{1}$ y perdida de la identidad de los territorios. Se trata de un cambio de paradigma a la clásica planificación del turismo (Cox 2009), ya que no son solamente el sector público ni privado los que lideran la actividad, sino son también las comunidades locales. A continuación se presenta una figura explicativa de las implicancias en la relación entre turismo de base comunitaria y gobernanza.

1 Compraventa de terrenos y bienes inmuebles por inversionistas que buscan obtener beneficios mediante la producción de espacio y fluctuaciones de precio. 
Figura 1. La gobernanza y el TBC

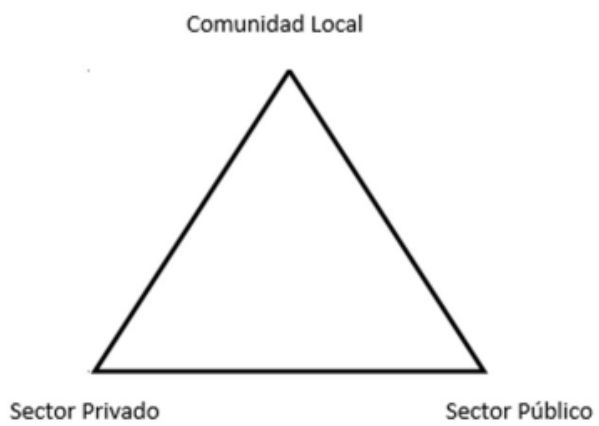

Se escapa de la clásica planificación turística en donde principalmente los esfuerzos recaían en tratar de coordinar al sector público con el privado, sin considerar a la comunidad local como un interlocutor válido a la hora de planificar el desarrollo turístico de un territorio o destino (Pacheco et. al. 2014).

Según Mayntz (1998), el origen de gobernanza proviene de un intento de la autoridad política por modelar las estructuras y los procesos socioeconómicos. Sus orígenes parten de la teoría del desarrollo entre las décadas de 1950 y 1960 alcanzando su auge a partir de mediados de la década del 90 (Barbini 2011).

Los sistemas de gobernanza en un principio comienzan con las alianzas público - privadas pero, sin embargo, se olvidan por un momento del tercer sector, el sector social. Según Ostrom (2000), los sistemas de organización pueden revertir los problemas de competencia e individualismo en los territorios, y no necesariamente la solución tiene que venir de terceros, como el estado o el sector privado principalmente. La cooperación permite niveles de autogestión para resolver problemas de manera eficiente por las propias comunidades (sector social) y manejar la información necesaria para autogestar cambios. En este sentido, el concepto de gobernanza ha venido paulatinamente incorporando a la comunidad local, ya que se viene visibilizando la necesidad de que los propios residentes logren empoderarse y participar de los procesos de toma de decisiones en las intervenciones territoriales que los afectan.

Mayntz (1998) da a reflexionar si realmente los sistemas de gobernanza son propiamente endógenos o autónomos en su gestión, y no modelos alternativos de un gobierno jerárquico. 
Se comprende que ambos fenómenos, gracias a la complejidad de las dinámicas humanas, confluyen, siendo la gobernanza un receptor de corrientes autónomas y endógenas, pero también responden a marcos institucionales provenientes de sistemas de gobiernos regionales y nacionales.

En este artículo, se comprende a la gobernanza como una alianza entre el sector público, privado y comunidad local, para hacer frente a problemas territoriales y desarrollar posibles soluciones, estrategias o políticas públicas. En el último tiempo se viene considerando a la sociedad civil en la gobernanza, ya que en su origen fue desarrollada por los gobiernos para que el sector privado sea un brazo en la gestión gubernamental, sobre todo en temas económicos, en donde las decisiones de las intervenciones a desarrollar en los territorios, eran tomadas por unos pocos desde arriba hacia abajo. Sin embargo, también surgen gobernanzas de corrientes endógenas, con una lógica de abajo hacia arriba que toma fuerza, aquí las soluciones a los problemas del desarrollo territorial son impulsadas por las propias organizaciones locales y/o comunitarias (Mayntz 1998, Pacheco et. al 2014).

\section{Figura 2. Direcciones de la gobernanza}

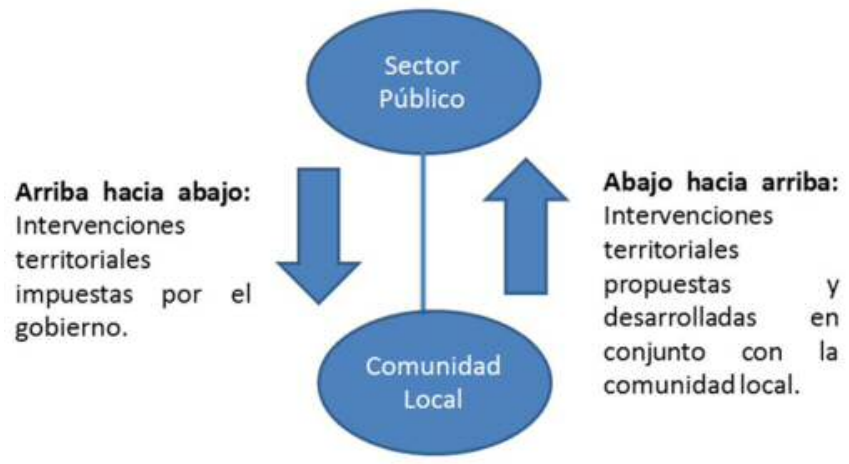

\section{IV- Los territorios de Panguipulli y el Turismo de Base Comunitaria}

El TBC surge en Latinoamérica y es impulsado, en una primera instancia, por comunidades indígenas, ya que conservan formas de organización comunitaria en aspectos económicos, culturales - ambientales etc., se desarrolla tanto en espacios rurales como urbanos, siendo un ejemplo, lo que acontece en Salvador de Bahía, Brasil, con la experiencia de turismo comunitario urbano en el barrio Cábula, que es liderado por comunidades afrodescendientes (Santos da Silva 2011). El turismo comunitario surge en el territorio comunal de Panguipulli, dado que en el sur de Chile desde el año 2000 en adelante emergen iniciativas en las que comunidades y organizaciones mapu 
che (huilliche, lafquenche, pehuenche) han liderado interesantes procesos, como por ejemplo, Mapu Lahual en la Provincia de Osorno, Llagüepulli en Lago Budi en la costa de la Araucanía, Cooperativa Ngen en Curarrehue, Trekaleyin en Alto Bio Bio y la experiencia de Quinquén en la comuna de Lonquimay.

Las particularidades territoriales de Panguipulli son muy similares a los lugares en donde se han levantado estos procesos, por lo que no cabe duda de que todas estas experiencias fueron influenciando para que en el territorio se comenzara a evaluar la posibilidad de potenciar al TBC, como una actividad productiva alternativa, pero con un claro énfasis en la conservación de la biodiversidad y de la identidad de las comunidades locales. Dentro de los primeros acercamientos de las comunidades sobre el concepto de turismo de base comunitaria, destacan los "5 Diálogos" (instancias de dialogo entre el sector público, privado, comunidad local y academia) efectuados en la comuna, más 2 seminarios internacionales efectuados en Chile y Brasil, liderados por el CEAM UACh e instituciones asociadas como por ejemplo la Incubadora Tecnológica de Cooperativas Populares (ITCP) de la Universidad Federal de Paraná (UFPR) de Brasil.

En la comuna de Panguipulli, surgen nuevas iniciativas de turismo con base local y comunitaria, por parte de comunidades que ven la posibilidad de conservar su identidad y naturaleza por medio de una actividad complementaria a los distintos papeles que deben atender en su tiempo de vida. Estas iniciativas, en muchas ocasiones responden a un alto grado de relación con la identidad territorial, como por ejemplo, las actividades de la Comunidad Inalafquen en Lago Neltume, aquí se viene realizando una Bajada Ancestral por el rio y humedal Cua Cua y por el lago Neltume, en defensa de las aguas y sus territorios aledaños que se verán intervenidos e inundadas áreas de importancia espiritual y cultural si se construye la central hidroeléctrica de Neltume por parte de la transnacional Endesa - Enel. Esta actividad es acompañada por una feria de productos locales, sirviendo para dinamizar la economía local, pero también para demostrar que las comunidades locales usan esos espacios para satisfacer sus necesidades humanas fundamentales en virtud de su calidad de vida, necesidades de subsistencia, recreación, libertad e identidad (Max Neef et. al. 2001). El TBC como una herramienta de acción social, es muy bien comprendido por algunas organizaciones de Panguipulli, como también por líderes locales como se manifiesta (N.C.) en el diario Uchile, el 19 de enero de 2014. "(El TBC) para nosotros es bastante importante porque nos da a conocer en el aspecto cultural. Le da otro enfoque, porque como que siempre se habla de los lugares o comunidades que están en «conflicto», así como «comunidades mapuche conflictivas». Entonces yo creo que ese es el ámbito que hay que cambiar. Que la gente comience a ver lo bueno que la gente tiene. En realidad, el mapuche no es así. El mapuche es más dinámico, quizás tiene mejor convivencia. Dejar de mostrar lo que siempre se muestra en la televisión, que si hay un mapuche es porque es conflictivo. En realidad, mu 
chas veces las invasiones de las grandes empresas generan que uno comience a defenderse, y simplemente por defenderse uno ya es «terrorista»", señaló" (Romero 2014).

Las acciones del Programa en la comuna de Panguipulli comienzan en el año 2013 con el proyecto "Nodo de Turismo Comunitario en Zona de Amortiguación del Parque Nacional Villarrica y Reserva Nacional Mocho Choshuenco". La comuna de Panguipulli se emplaza dentro de una zona de amortiguación entre dos áreas protegidas con alto valor de conservación para la diversidad biocultural, en donde comunidades mapuche habitan en armonía con el paisaje. Dada su particularidad comunal de contar con dos áreas protegidas, el Programa cuenta con investigadores y estudios en materias de prácticas productivas, turismo comunitario, conservación, agricultura familiar, cultura e identidad y derechos humanos. Se trata de un Programa abordado desde la interdisciplina hacia la transdisciplina, con claro enfoque en los problemas tomando elementos de la investigación acción participativa para generar espacios comunitarios de reflexión sobre los fenómenos socioambientales.

Panguipulli es una comuna grande en términos de superficie, una de las más grandes de Chile y la más grande de la región de Los Ríos. Por lo tanto, se trata de un territorio extenso compuesto por micro territorios con realidades similares pero con cotidianidades diferentes. Según el Censo (2002) Panguipulli cuenta con $3.292 \mathrm{~km}^{2}$ de superficie y 33.723 habitantes, igual a una densidad de 10 habitantes por $\mathrm{km}^{2}$, con una población masculina del 51,27\% y una población femenina del 48,73\%. Un 52,25\% de los habitantes vive en espacios rurales mientras que un $47,75 \%$ habita en espacios urbanos. Un 30,8\% de la población en Panguipulli declara ser mapuche, con un número igual a 10.264 personas que equivalen al $25,3 \%$ de la población mapuche de la región de Los Ríos (Censo 2002; Hasen et. al. 2014). Es claramente visible que un poco más de la mitad de las personas que vive en la comuna de Panguipulli lo hace en espacios rurales, así mismo al menos un tercio de la población es mapuche. El Programa se desarrolla en 8 territorios dentro de la comuna de Panguipulli, que se muestran a continuación en el siguiente mapa. 


\section{Mapa 1. Territorios en investigación sobre el Turismo de Base Comunitaria de la comuna de Panguipulli}

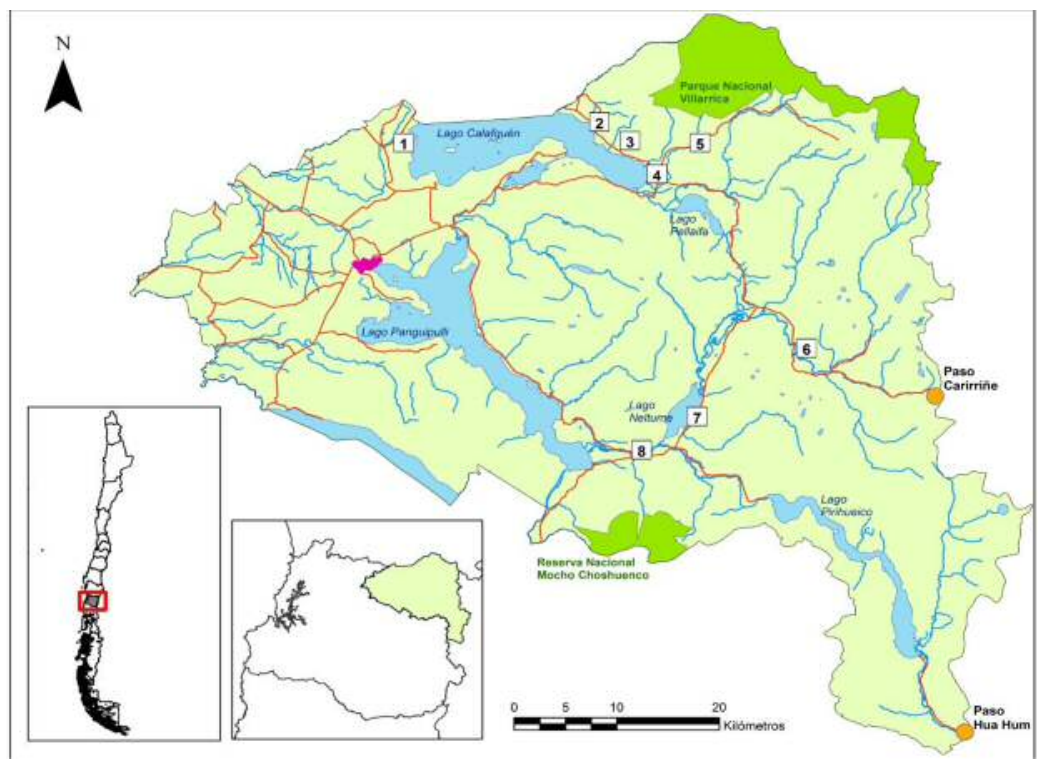

En el número 1 del mapa, el territorio "Huitag" es un antiguo asentamiento mapuche, que cuenta con un humedal en la costa oeste del lago Calafquen. Actualmente se encuentra asociada al Programa la Comunidad Indígena Cacique Llancapán. Cuentan con el acceso al humedal de Huitag, sitio de importancia ecológica.

En el número 2 del mapa, el territorio "Pucura" es un antiguo asentamiento y lof mapuche, su nombre significa dos piedras en mapudungun $(\mathrm{Epu}=\mathrm{dos}$, Kurra $=$ piedra), se trata de uno de los lugares en donde se conservan aún sitios patrimoniales y arqueológicos que enriquecen la historia y origen del pueblo mapuche. Actualmente existe una experiencia de economía solidaria la "Agrupación de Pequeñas Artesanas y Agricultoras de Pucura", que cuentan con una Feria de Productos Locales en temporada estival, esta iniciativa es liderada por un grupo de mujeres mapuche. El lof mapuche de Pocura colinda con la parte sur del Parque Nacional Villarrica.

En el número 3, el territorio "Traitraico", colinda por el noreste con la localidad de Pucura, se ubica entre el lado norte del lago Calafquen y las faldas del Volcán Villarrica, esta localidad colinda con el Parque Nacional Villarrica. Actualmente la comunidad Felipe Caripan, está potenciando algunas iniciativas de turismo de base comunitaria, sin embargo presentan problemas para su desarrollo, tales comola especulación inmobi 
liaria, algunos espacios defendidos por la comunidad han tendido a ser blanco de conflictos como desalojos, destrucciones de rucas e incendios de viviendas (Durán 2013).

En el número 4, el territorio "Los Cajones" es un sector cercano al Parque Nacional Villarrica. Su acceso más importante parte desde Coñaripe camino al P.N. Villarrica por un camino de $24 \mathrm{~km}$ aprox. En este sector habitan residentes locales que descienden de mapuche y colonos chilenos, que se asentaron entre un cordón de cerros repletos de bosque nativo que rodean al volcán Villarrica. En este sector existen una variedad de fuentes termales, dando origen al menos a 3 reconocidos centros termales.

En el número 5, el territorio "Coñaripe", su nombre significa "sendero del guerrero" en mapudungun, se ubica al lado este del lago Calafquén con un balneario que lo caracteriza, es un antiguo pueblo maderero, y actualmente es un lugar visitado masivamente en verano por turistas nacionales provenientes de centros emisivos tales como la Región Metropolitana, Valparaíso, Concepción y Temuco. El pueblo actual tuvo que ser reconstruido luego de la erupción del volcán Villarrica en 1964, que destruyó al 50\% del poblado de Coñaripe. Actualmente cuenta con una oferta turística, especialmente iniciativas de alojamiento (cabañas).

En el número 6, el territorio "Liquiñe" se emplaza en su valle homónimo, es una localidad de tradición maderera, sin embargo existen grandes extensiones de bosque nativo renoval en los cerros actualmente que hacen de su entorno un paisaje armónico. Actualmente la producción maderera ha mermado y se desarrollan actividades productivas silvoagropecuarias, turísticas (reconocido por sus termas, pesca y recientemente por sus senderos), artesanías (lana y madera) y comercio local. En Liquiñe existen organizaciones sociales y productivas con estrecha relación con el turismo comunitario y economía solidaria, que actualmente participan del Programa, estas organizaciones corresponden a: Agrupación de Mujeres Manos de la Cordillera de Liquiñe, la Agrupación de Empresarios Turísticos Puwinkul de Liquiñe y la Asociación de Turismo Ecológico y Guías de Liquiñe Trekantun.

El número 7, territorio "Lago Neltume", se localiza al sur de Coñaripe y al suroeste de Liquiñe, es un territorio mapuche que es habitado por comunidades, en el Programa actualmente participa la Comunidad Inalafquen. Su nombre se debe al hermoso lago que actualmente protagoniza uno de los conflictos socioambientales más importantes de la Región de Los Ríos. El paisaje es influenciado por el imponente volcán Mocho Choshuenco.

El número 8, el territorio "Punahue", se localiza al sur de Liquiñe, en este lugar se encuentra la comunidad mapuche Manuel Curilef quienes a través de una agrupación de mujeres organizan la feria local. Colindan con la Reserva Nacional Mocho Choshuenco, la cual es una área en la que el sector público ha declarado potenciar a nivel turístico. 
Por cierto, el Plan Maestro para el desarrollo turístico de la Reserva Nacional Mocho Choshuenco, contempla senderos a los glaciares que pasan por sobre la propiedad indígena de la comunidad Manuel Curilef (CONAF 2013).

\section{V- Metodología}

\section{1- Aplicación de Técnicas de la Investigación Acción Participante:}

Desde el 2013 fueron realizados distintos talleres, diálogos y seminarios para dialogar sobre el concepto de turismo de base comunitaria entre las comunidades locales, con invitación de expertos internacionales. Se realizó un mapeo participativo sobre usos ancestrales de las comunidades mapuche en zonas aledañas y dentro del Parque Nacional Villarrica. Así también fueron mapeados los intereses futuros sobre estos espacios de uso ancestral, por parte de 15 personas adultas (mayores de 30 años) pertenecientes a las organizaciones, comunidades y familias mapuche que desarrollan iniciativas de turismo de base comunitaria, y que habitan zonas aledañas del Parque Nacional Villarrica en los territorios de Pucura, Traitraico y Los Cajones.

\section{2- Generación de Mesas de Discusión Interdisciplinaria:}

Entre marzo y diciembre de 2014, fueron realizadas reuniones de trabajo en marco del Programa, en donde se generaron discusiones entre distintos investigadores de las áreas: sociales, económicas y ambientales sobre resultados preliminares tales como: la especulación inmobiliaria, los megaproyectos hidroeléctricos, el asistencialismo, el sobreintervencionismo, la coaptación ${ }^{1}$ por agentes públicos y privados, el sistema de certificación de calidad y la pérdida de la identidad, concesiones en áreas protegidas, formalización, desarrollo productivo y sustentabilidad etc. Estas discusiones fueron retroalimentando las distintas problemáticasdelturismodebasecomunitariaidentificadascomoresultadosdeinvestigación.

\section{3- Revisión de Archivos sobre la Experiencia local y Latinoamericana:} Se realizó una revisión de investigaciones que dan cuenta de distintas experiencias de turismo de base comunitaria. Así también se revisaron distintas publicaciones sobre las relaciones entre gobernanza y el turismo de base comunitaria, sus particularidades y similitudes. Para esto se utilizaron distintas publicaciones de libros, artículos de revistas académicas y tesis. Se revisaron archivos audiovisuales, notas de campo y otros documentos con información relevante sobre el TBC en Panguipulli.

$\overline{1}$ Coaptación: Según la Real Academia Española (RAE 2016), la palabra "coaptar" significa "Proporcionar, ajustar o hacer que convenga algo con otra cosa". Proviene del latín coaptare, en donde aptare significa adaptar. Por lo tanto, el concepto alude a adaptar algo junto a un otro. Es un concepto utilizado en publicaciones relacionadas a los movimientos sociales, para explicar fenómenos de alineación de los intereses de ciertos grupos sociales sobre otros, a través de asimetrías de poder económico y sociopolítico. Como sucede con el Estado y las grandes empresas, que, mediante sus propios intereses y mecanismos de control, inducen e influencian los intereses de las organizaciones sociales. 


\section{VI-Principales Problemáticas del Turismo de Base Comunitaria (TBC) en Panguipulli}

Fueron identificadas y caracterizadas distintas problemáticas del TBC en Panguipulli según temáticas analizadas en mesas de discusión interdisciplinaria realizadas en marco al Programa de Turismo de Base Comunitaria, Economía Solidaria y Desarrollo de Base Local, estas corresponden a:

\begin{tabular}{|c|c|c|}
\hline 1 & $\begin{array}{l}\text { Co-Administración de } \\
\text { Áreas Protegidas }\end{array}$ & $\begin{array}{l}\text { Necesidad de participación de las comunidades rurales y mapuche en la administración y } \\
\text { gestión de áreas protegidas y zona de amortiguación. }\end{array}$ \\
\hline 2 & $\begin{array}{l}\text { Propiedad indigena, } \\
\text { rural y/o familiar }\end{array}$ & Necesidad de regular los derechos de propiedad de las comunidades rurales e indigenas. \\
\hline 3 & $\begin{array}{l}\text { Especulación } \\
\text { inmobiliaria } \\
\end{array}$ & Necesidad de proteger las propiedades de las comunidades rurales e indigenas. \\
\hline 4 & $\begin{array}{l}\text { Conflictos } \\
\text { socioambientales }\end{array}$ & $\begin{array}{l}\text { Necesidad de proteger territorios de megaproyectos que afectan el desarrollo de base } \\
\text { local y comunitario. }\end{array}$ \\
\hline 5 & $\begin{array}{l}\text { Coaptación e } \\
\text { intervención }\end{array}$ & Necesidad de autogestar la autonomía de las organizaciones. \\
\hline 6 & Formalización & Necesidad de regularizar la formalización de las iniciativas del Circuito. \\
\hline 7 & Habilidades endógenas & $\begin{array}{l}\text { Necesidad de empoderar a la comunidad de sus saberes y habilidades, como tambièn } \\
\text { incorporar conocimientos técnicos y cientificos complementarios. }\end{array}$ \\
\hline 8 & $\begin{array}{l}\text { Turismo masivo y de } \\
\text { enclave }\end{array}$ & $\begin{array}{l}\text { Evitar la transformación de los territorios en destinos masivos con grandes impactos } \\
\text { socioambientales. }\end{array}$ \\
\hline 9 & $\begin{array}{l}\text { Invisibilización de la } \\
\text { oferta informal }\end{array}$ & $\begin{array}{l}\text { Necesidad de visibilizar la oferta de TBC que se encuentra olvidada y escondida por ser } \\
\text { informal y no responder a todos los requerimientos de formalización. }\end{array}$ \\
\hline 10 & $\begin{array}{l}\text { Acceso al ahorro, } \\
\text { crédito e inversión }\end{array}$ & Necesidad de acceso a fuentes de financiamiento para la inversión de las iniciativas. \\
\hline 11 & Identidad & $\begin{array}{l}\text { Necesidad de proteger y rescatar modos de vida, tradiciones y cotidianidades } \\
\text { territoriales. }\end{array}$ \\
\hline
\end{tabular}

Fuente: Pacheco 2015, CEPIAL Osorno.

En este artículo se describirán los resultados asociados a tres de estas problemáticas que dan cuenta de la relación entre el turismo de base comunitaria y los procesos de gobernanza en el territorio en cuestión. Estas corresponden a 1- Co- administración de áreas protegidas, 2- Conflictos Socioambientales y 3- Especulación inmobiliaria. 


\section{1- Co-Administración de Áreas Protegidas y Zonas Aledañas por comunidades mapuche:}

Los Parques y reservas fueron creados en donde existían comunidades viviendo por muchos años, lo que ha conllevado a una serie de prohibiciones y conflictos, respecto a los territorios ancestrales de comunidades mapuche que se encuentran dentro de las áreas protegidas y en zonas adyacentes, los actuales descendientes cuentan con derechos consuetudinarios sobre estos espacios (Sansolo 2009; Guerra 2014; Aylwin \& Cuadra 2011).

En Brasil históricamente se ha alejado a las comunidades de los procesos de gobernanza de las áreas protegidas (Bannerman 2001, Irving 2001, Ferreira de Matos 2009). Lo mismo sucede en Chile y en la comuna de Panguipulli, en donde actualmente no existe el respaldo legislativo ni normativo para impulsar sistemas de administración, gestión o manejo integrado de territorios ancestrales por parte de comunidades mapuche dentro de áreas protegidas, tal como ya sucede, por ejemplo, en la Amazonía boliviana con comunidades indígenas en el Parque Nacional Madidi.

Se ejecutó un taller denominado "Turismo Comunitario y Concesiones Administrativas en Aéreas Protegidas del Estado: Una mirada del derecho internacional de los derechos humanos", en Panguipulli, el 30 de julio de 2014 en marco al proyecto NODO de Turismo Comunitario, participaron 15 personas, mayores de 30 años que cuentan con iniciativas en territorios cercanos al Parque Nacional Villarrica en la comuna de Panguipulli, se realizó un mapeamiento participativo con objeto de identificar aspectos negativos y positivos sobre la actual administración del parque. Estos fueron los argumentos de los miembros de organizaciones comunitarias sobre el fenómeno de gobernanza en el P.N. Villarrica.

Figura 4. Aspectos negativos

\begin{tabular}{|l|}
\hline \multicolumn{1}{|c|}{ Aspectos negativos } \\
\hline Perdida de propiedad de las comunidades ancestrales. \\
\hline Problemas de acceso al P.N. Villarrica y cosecha de productos de recolección (piñones). \\
\hline Abandono y quemas de ranchas de las comunidades dentro del P. N. Villarrica. \\
\hline Los senderos de las pinaladas se encuentran descuidados. \\
\hline
\end{tabular}

Los representantes de las iniciativas y organizaciones que participan del Programa, identificaron una pérdida de propiedad de las comunidades ancestrales, ya que el área del P.N Villarrica se encuentra dentro de tierras mapuche. No sólo eso ha llevado a un problema sobre la pérdida de la propiedad, sino también el derecho a uso de esos espacios, que eran usados antiguamente, como, por ejemplo, para las "piñonadas o pinaladas", en donde existen antiguos senderos que conducen a las araucarias, los que se actualmente encuentran descuidados. Las araucarias (araucaria araucana) entregan el fruto del piñón que es parte fundamental de la dieta de comunidades mapuche que 
habitan en zonas cercanas a la Cordillera de los Andes en el sur de Chile.

Antiguamente las familias mapuche acostumbraban habilitar en verano ranchos para vivir cercanos a las araucarias dentro del actual Parque, lo que se denomina como "veranada". Estos ranchos fueron siendo destruidos, quemados y olvidados en el tiempo.

Actualmente, la administración del Parque Nacional Villarrica es ejecutada por la Corporación Nacional Forestal (CONAF), organismo de derecho privado dependiente del Ministerio de Agricultura. Desde de la habilitación de una entrada al P. N Villarrica desde la región de Los Ríos por la comuna de Panguipulli, se han impulsado una serie de regulaciones sobre el Parque. Lo que ha conllevado a una percepción negativa sobre la administración del Parque, su participación en la planificación y regulaciones, como también una falta de interés por parte del sector público de integrar a las comunidades mapuche y rurales adyacentes al Parque.

Figura 5. Aspectos positivos

\begin{tabular}{|l|}
\hline \multicolumn{1}{|c|}{ Aspectos positivos } \\
\hline Posibilidad de conservar el patrimonio y biodiversidad a través del turismo. \\
\hline El turismo como una alternativa de renta familiar. \\
\hline Evitar la inserción de grandes proyectos turisticos de enclave dentro del Parque. \\
\hline $\begin{array}{l}\text { Interés por la instalación de refugios comunitarios para el desarrollo de productos de turismo de base } \\
\text { comunitaria dentro del Parque. }\end{array}$ \\
\hline El turismo de base comunitaria como una actividad productiva complementaria, y no un fin en si mismo. \\
\hline Politicas públicas del Estado para el fomento del TBC en áreas protegidas y zonas aledarias. \\
\hline
\end{tabular}

Los aspectos positivos sobre la percepción de los participantes de las iniciativas de turismo de base comunitaria de los territorios, apuntan finalmente a la posibilidad de impulsar un sistema de gobernanza local, en donde comunidades y organizaciones de residentes locales, en su mayoría mapuche, logren obtener el derecho de uso y administración de un espacio dentro y adyacente al área protegida, que permita seguir fomentando la conservación de la naturaleza como también generar renta de base local en armonía con el paisaje.

El turismo de base comunitaria es una herramienta para evitar la inserción de grandes proyectos turísticos de enclave dentro del Parque, que no son un fomento para la economía local ya que no consideran la participación ni beneficio de las comunidades mapuche adyacentes a las áreas protegidas, actualmente estas comunidades cuentan con iniciativas que no superan los 24 mil dólares norteamericanos de ventas anuales. Los proyectos turísticos dentro de las áreas protegidas deben incorporar a la comunidad local, no solamente en la mano de obra, sino también en su participación activa en la gestión de espacios para la conservación y recreación. 
Respecto a las comunidades mapuche, se debe respetar el Convenio 169 de la OIT, por lo tanto, cualquier proyecto que afecte terrenos de propiedad indígena, o simplemente territorios de uso ancestral, deben ser efectuadas por la institucionalidad pública las pertinentes consultas a las comunidades involucradas (Guerra 2014).

Finalmente, los participantes mencionaron el interés por instalar refugios comunitarios dentro del Parque para gestar vivencias de turismo de base comunitaria, tal como sucede en otros países Latinoamericanos, como Bolivia en el Parque Nacional Madidi, allí existen experiencias de Áreas de Manejo Integrado para el desarrollo del turismo de base comunitaria por comunidades amazónicas.

\section{2- Conflictos socioambientales}

Se identifica una producción de espacio por parte del sector Privado y el Estado que conlleva al desarrollo desigual en áreas protegidas y lugares de importancia ecosistémica y cultural (hidroelectricidad a pequeña y gran escala, concesiones mineras), megaproyectos generan impactos negativos a las comunidades locales, tal como la extracción de petróleo en el Amazonas y las intenciones de instalar una Central Hidroeléctrica, por parte de la Transnacional Endesa - Enel, en el territorio de Lago Neltume, Panguipulli, Chile.

En la región de Los Ríos, y más precisamente en la comuna de Panguipulli aún existen latentes actuales proyectos de hidroelectricidad. Así, por ejemplo, es lo que sucede en Liquiñe, Lago Neltume y Punahue, con el proyecto de instalación de la Central Hidroeléctrica de la transnacional Endesa - Enel. Este proyecto hidroeléctrico ha sido cuestionado por las comunidades mapuche entre ellas la comunidad Inalafquen, sobre los efectos de inundación de espacios sagrados (cancha del nguillatwe) y desplazamiento de los asentamientos humanos que se encuentran cercanos al lago Neltume. Asimismo, una serie de otros impactos, como la generación de caminos para levantar torres de alta tensión, en sectores que unen a las localidades de rio Liquiñe, Rio Hueico, Lago Neltume y Punahue. Actualmente las comunidades y sus organizaciones se acogen al Convenio 169 de la OIT, para reivindicar derechos, pero sin embargo aún los resultados no son rotundos en cuanto al término oficial de las obras en este territorio. El caso de la central Pullinque en la comuna es un ejemplo concreto, construida en la década del 70 y operativa desde entonces, la energía eléctrica llega a las comunidades de Pucura y Traitraico a mediados de los 90, a los Cajones a principios del 2000 y hay sectores como el caso de Cachim que aun no cuenta con energía eléctrica pese a estar a menos de $80 \mathrm{~km}$ de la central. 
Los argumentos clásicos sobre el progreso y la generación de trabajo, no son argumentos reales sobre los beneficios de los megaproyectos hidroeléctricos, por ejemplo, para la construcción demandan centenares de obreros, por periodos cercanos a los 5 años, mientras que luego solo quedan trabajando menos de 2 decenas de técnicos, que generalmente son personas de fuera de los territorios. Realmente son muy pocos los beneficios a la calidad de vida de las comunidades locales, poco acceso a la renta, impactos al medioambiente, pérdida de identidad, pérdida de sitios patrimoniales, patologías sociales, transformación negativa del paisaje etc. Cualquier alteración al paisaje, no es solo un impacto para quienes habitan, sino también para quienes visitan, según el CEAM UACh (2014) el 77\% de las personas que visita Panguipulli no volvería si se produjeran alteraciones al paisaje de lagos, ríos, volcanes y bosques. Esto también se traduce en un impacto significativo al sector turismo, a las iniciativas de turismo de base local y comunitaria del territorio que generan un importante aporte a la renta familiar sobre todo en temporada estival. Megaproyectos como este, son incompatibles con el turismo de base local, ya que impactan el entorno en donde se desenvuelve la actividad (Pacheco \& Smulewicz 2013). Se trata de vivencias que se basan en la relación entre el patrimonio natural y sociocultural presente en el territorio. Destaca, por ejemplo, la Bajada Ancestral de Lago Neltume, que se realiza todos los años en el mes de febrero, actividad de reivindicación en defensa de la libertad de las aguas y el derecho de las comunidades mapuche y rurales en territorios con conflicto de uso. Esta actividad es organizada por la Comunidad Mapunche Inalafquen, con objeto de visibilizar los impactos de la transnacional Endesa - Enel por la construcción de una hidroeléctrica en Lago Neltume, comuna de Panguipulli. El impacto de la transnacional y su intervención en el territorio ha sido tan nefasto, que por efecto de esto se generó la división de la comunidad ancestral Juan Quintuman, formándose en 2010 la comunidad Inalafquen, que se descuelga de la comunidad ancestral, debido a su posición contraria a Endesa y su plan de asistencialismo.

No son solamente el Estado, el mercado y los turistas los que producen los espacios, sino también las sociedades que habitan esos espacios, algunos convertidos y/o reemplazados por emprendedores turísticos o actuando en una contra racionalidad a las determinaciones hegemónicas (Burstyn et. al 2009:98). Sin duda, el papel de la sociedad, de la comunidad local en los territorios, se hace fundamental a la hora de defender los espacios de uso público y bien común, como también para usar al turismo como una herramienta para fortalecer la identidad, el rescate patrimonial y las oportunidades para la generación de renta de base local. Así lo viene realizando por ejemplo la Comunidad Mapunche Inalafquén, no se han convertido en emprendedores turísticos per se, sino cuentan con una iniciativa comunitaria y económicamente solidaria (feria de productos locales y "Bajada Ancestral Lago Neltume"). 


\section{3- Especulación inmobiliaria:}

El turismo masivo de sol y playa genera una transformación de los espacios y sujetos locales, por lugares de confort para especuladores, segundos residentes y turistas. (Burztyn, Bartholo y Delamaro 2009; Coriolano 2007; Oliveira 2009; Pacheco et. al. 2011).

Las iniciativas de turismo de base comunitaria participantes del Programa consideran que el desarrollo turístico no debe ser explosivo como resultó ser en Pucón (Pacheco et. al. 2011). En la comuna de Panguipulli como en muchas comunas en donde se desarrolla el turismo, se generan especulaciones inmobiliarias sobre todo en espacios de gran interés turístico, como por ejemplo la ribera del lago Calafquén en los sectores de Coñaripe, Traitraico, Pucura y Huitag. Actualmente se dan algunos conflictos de propiedades protagonizados entre inversionistas con intereses financieros y habitantes rurales y mapuche del territorio.

Con el TBC es posible conservar modos de vida, evitar la transformación de los espacios donde habitan y el desplazamiento de las comunidades locales por ventas de propiedad (Mendonça 2009; Henríquez 2010, Sampaio 2005). En Prainha do Canto Verde, ubicada en el municipio de Beberibe, litoral este del Estado del Ceará, Brasil, a 126 km de la capital de Fortaleza, se desarrolló un proyecto de turismo considerado como una solución local en busca de alternativas a un sistema caracterizado por la crisis de la pesca, la especulación inmobiliaria, por la expulsión de residentes locales de sus tierras y por la falta de participación efectiva de los residentes en la toma de decisiones sobre el territorio. Las primeras discusiones sobre el turismo con la comunidad, se desarrollaron desde 1993, cuando a pesar de algunas resistencias los habitantes se percataron que la llegada del turismo era inevitable. En 1994, se realizó una investigación en marco del "Proyecto Turístico Socialmente Responsable" de Prainha do Canto Verde, su objetivo era conocer la opinión de los habitantes sobre la llegada del turismo a Prainha. Los habitantes mencionaron que uno de los principales aspectos positivos era la generación de renta y trabajo, y en sus aspectos negativos mencionaron la desconfianza por la explotación del lugar por agentes externos y la venta de las tierras (Mendonça 2009).

Las primeras discusiones sobre turismo de base comunitaria en la comuna de Panguipulli se desarrollan en 2013 impulsado por el CEAM UACh, Bosque Modelo Panguipulli y la Ilustre Municipalidad de Panguipulli, más el apoyo de comunidades y organizaciones vinculadas al turismo de base comunitaria y local. En el momento en que la comunidad local reflexiona acerca de que el turismo de sol y playa ya era una realidad con años de desarrollo, se plantean el desafío de que la actividad pueda ser desarrollada en beneficio de la comunidad. 
A las mujeres de Pucura se les preguntó sobre qué era para ellas el turismo comunitario, ellas respondieron: Es trabajar en comunidad, en grupo con un objetivo común. Una forma de trabajar en comunidad, y mostrar a otros su cultura.

Esperamos que respeten como somos y vivimos. No queremos gente avasalladora, que trate mal y que no le importe el medio ambiente. Con esa gente irrespetuosa no nos gusta trabajar. Nosotras y la gente mapuche en general, no piensa solo en ganar dinero, sino también en el kume mögnen, el buen vivir, que es importante para estar bien (M.LL.). Otras mencionaron: Es como recibir a un amigo... Nosotras atendemos a la gente con cariño y con calma.... Queremos que la gente se vaya contenta con lo que tenemos... (Proyecto NODO de Turismo Comunitario 2014, CEAM - UACh).

\section{VI- Conclusiones}

El turismo es una actividad productiva moderna en la que se reproduce una organización desigual y combinada de territorios capitalistas absorbiendo modos de vida y de producción de base local. Surge así la contradicción en las relaciones de apropiación del territorio para uso turístico (Coriolano, 2007). En esta dirección los escenarios locales, como los territorios del Programa, son testimonio real y verídico de conflictos y tensiones entre comunidades locales y la manera capitalista de apropiación del espacio donde los primeros ven un medio de vida y de continuidad de la existencia de su cultura, y la segunda que usa y se apropia del espacio y la naturaleza con ánimo de lucro.

En cuanto a la organización de la actividad turística existen dos modalidades, una que se inspira en la mirada clásica que es de arriba hacia abajo y otra alternativa que es la que va de abajo hacia arriba. Para el caso del TBC su inspiración y motivación se encuentran en la segunda opción, esto es, de abajo hacia arriba. Esto implica un ejercicio de escuchar y comprender el papel que juegan las y los residentes locales en la planificación y gestión de la actividad turística en los desafíos actuales. Para ello se contestan las definiciones clásicas del turismo como la entregada por el OMT que pone énfasis en el turista, o sea, en quien visita y olvidando y dejando en la subalternidad de la toma de decisiones a quienes reciben. Esto implica como se menciona en el cuerpo del artículo un cambio de paradigma en la forma de planificar y organizar la actividad turística.

El caso del Programa en la comuna de Panguipulli es ilustrador en la manera de organizar la actividad turística desde una visión de abajo hacia arriba, aquí la gobernanza propuesta surge de las inquietudes y visiones que las propias comunidades ven y proyectan de la actividad turística en su territorio para el futuro. Esta experiencia brinda la posibilidad de comprender el turismo de otro modo como un sendero 
interdisciplinar e intercultural que posibilita un recorrido por y para los actores locales recobrando el sentido de lo humano de esta actividad, pero con un respeto profundo por los otros seres que habitan en el territorio. Es una invitación a cruzar a la vereda de enfrente y ver cómo se organiza y desarrolla la actividad turista cuando se mira hacia al otro lado. Es también una invitación a colocar los pies en el barro y aprender que el conocimiento no solo está en la universidad, sino que también en la diversidad y riqueza de las comunidades tradicionales, y que no necesariamente es el experto el llamado a categorizar, planificar y organizar la actividad turística. En otras palabras, es una invitación a una discusión que se abre a la co-construcción y al diálogo de saberes.

\section{VII- Bibliografía}

Aylwin, J., Cuadra, X. (2011). Los desafíos de la conservación en los territorios indígenas en Chile, Observatorio de Derechos de los Pueblos Indígenas, Temuco.

Bannerman, M. 2001. Mamirauá: um guia da história natural da Várzea Amazônica. Tefé: IDSM.

Barbini, B.; Biasone, A.; Cacciutto, M.; Castellucci, D.; Corbo, Y., y Roldán, N. (2011). Gobernanza y turismo: Análisis del estado de arte. Simposio Internacional de Gobernanza y Cambios Territoriales: Experiencias Comparadas de Migración de Amenidad en las Américas. Pucón, Chile.

Bursztyn, I., Bartholo, R., Delamaro, M. (2009). Turismo para quem? Sobre caminhos de desenvolvimento e alternativas para o turismo no Brasil em Bartholo, R., Sansolo, D.G, Bursztyn, I. (Orgs em Bartholo, R., Sansolo, D.G, Bursztyn, I. (Orgs.). Turismo de base comunitária: Diversidade de olhares e experiências brasileiras. Rio de Janeiro (RJ), Letra e Imagem, pp.302-318).

CEAM UACh. (2014). Resultados Proyecto "Nodo de Turismo Comunitario en Zona de Amortiguación del Parque Nacional Villarrica y Reserva Nacional Mocho Choshuenco". Cofinanciado por la Corporación de Fomento Productivo (CORFO), Valdivia, Chile.

Coriolano, Luzia Neide. (2007). Turismo e Natureza. Fortaleza: EDUECE, Brasil. Corporación Nacional Forestal (CONAF). 2013. Plan Maestro Parque Nacional Villarrica, Chile.

Cox A. R. (2009). Turismo indígena y comunitario en Bolivia. Un instrumento para el desarrollo socio-económico e intercultural. La Paz Bolivia: Plural editores.

Durán, M. (2013). Panguipulli, Traitraico: Vuelve el despojo territorial a tierras mapuche. http://www.elpuelche.cl/?p=3722. El Puelche, 13 de diciembre de 2013.

Ferreira de Matos, F. (2009). "Ecoturismo e inclusão social na Resex Marinhado Delta do Parnaíba (MA/PI) tendências, expectativas e possibilidades", em Bartholo, R., Sansolo, D.G, Bursztyn, I. (Orgs em Bartholo, R., Sansolo, D.G, Bursztyn, I. (Orgs.). Turismo de base comunitária: Diversidade de olhares e experiências brasileiras. Rio de 
Guerra, F., Gómez, M. (2014). Informe "Análisis crítico de los modelos de gestión indirecta en áreas silvestres protegidas del estado: un estudio desde el derecho administrativo y el derecho internacional para la propuesta de un modelo de cogestión en el contexto del desarrollo turístico comunitario". Proyecto "Nodo de Turismo Comunitario en las zonas de amortiguación del Parque Nacional Villarrica y Reserva Nacional Mocho Choshuenco comuna de Panguipulli”. CEAM - UACh, Valdivia, Chile.

Hasen, F. (2014). Caracterización territorial sociocultural. Proyecto "Nodo de Turismo Comunitario y Economía Solidaria de la zona de amortiguación del Parque Nacional Villarrica y la Reserva Nacional Mocho Choshuenco". CEAM-UACh, Valdivia. Henríquez, C.; Zechner, T.; Sampaio, C. (2010). Turismo y sus Interacciones en las Transformaciones del Espacio Rural. Revista Ciencias Sociales. Universidad Austral de Chile.

Instituto Nacional de Estadísticas (INE). Censo 2002. Resultados Población y Vivienda: País-Región-Provincia-Comuna.

Irving, M. A. (2001). Construindo um Modelo de Planejamento Turístico de Base Comunitária: Um Estudo de Caso. Série Documenta, v. 7, n. 10, p. 59-82

Max-Neef, M.; Elizalde, A. y Hopenhayn, M. (2001). Desarrollo a escala humana. Editorial Nordan-Comunidad. Montevideo, Uruguay.

Mayntz, R. (1998). Nuevos desafíos de governance. Traducción: "New Challenges to governance teory". Jean Monet Chair Paper RSC N. 98/50.

Mendonça, T. C. de M. (2009). "Turismo socialmente responsável da Prainha do Canto Verde: uma solução em defesa do local herdado". In: Bartholo, R.; Sansolo, D. G. \& Bursztyn, I. (Orgs.) Turismo de base comunitária: diversidades de olhares e experiências brasileiras. UFRJ, Letra e Imagem, Rio de Janeiro, pp. 289-301.

Oliveira Campos, A. (2009). Turismo e população dos destinos turísticos um estudo de caso do desenvolvimento e planejamento turístico na Vila de Trindade - Paraty/RJ; em Bartholo, R., Sansolo, D.G, Bursztyn, I. (Orgs em Bartholo, R., Sansolo, D.G, Bursztyn, I. (Orgs.). Turismo de base comunitária: Diversidade de olhares e experiências brasileiras. Rio de Janeiro (RJ), Letra e Imagem, pp.302-318).

Organización Mundial del Turismo (OMT). (2015). Glossary of tourism terms. https://s3-eu-west-1.amazonaws.com/staticunwto/Statistics/Glossary+of+terms.pdf Ostrom, E. (2000). El gobierno de los bienes comunes. La evolución de las instituciones de acción colectiva. México, UNAM-CRIM-FCE, 395p.

Pacheco, G.; Henríquez C.; Sampaio, C., y Oyarzún, E. (2011). Encadenamientos Socioproductivos y Ecosocioeconomía de las Organizaciones: Análisis propositivo para el Turismo de Intereses Especiales (TIE) en el territorio lacustre de la Región de La Araucanía. Gest. Tur. (Valdivia), dic. 2011, Nº. 16, p. 49-68. ISSN 0718-6428. 
Pacheco, G. (2015). Turismo de Base Comunitaria y procesos de gobernanza en la comuna de Panguipulli: Un análisis comparativo con la experiencia brasileña. En IV Congreso de Cultura y Educación para la Integración de América Latina - "el Buen Vivir". Organizado por el Programa ATLAS y Centro de Estudios de Desarrollo Regional y Local (CEDER) de la Universidad de Los Lagos, Chile, junto con Red CASLA CEPIAL y la Fundación para la Superación de la Pobreza.

Pacheco Habert, G.; Szmulewicz Espinoza, P. (2013). Sinergias y Conflictos entre desarrollo turístico y otros sectores económicos. El caso del Turismo de Intereses Especiales en la región de los Ríos. Gestión Turística, núm. 20, julio-diciembre, pp. 39-59 Universidad Austral de Chile Valdivia, Chile.

Romero, P. (2014). Nodo de Turismo Comunitario colabora en Feria Mapuche de Lago Neltume. Diario Uchile, 19 de enero. Disponible online: (http://radio.uchile. cl/2014/01/19/nodo-de-turismo-comunitario-colabora-en-feria-mapuche-de-lago-neltume).

Sansolo, D.; Burztyn, I. (2009). "Turismo de base comunitária: potencialidade no espaço rural brasileiro", em Bartholo, R., Sansolo, D.G, Bursztyn, I. (Orgs.). Turismo de base comunitária: Diversidade de olhares e experiências brasileiras. Rio de Janeiro (RJ), Letra e Imagem, pp. 142-161.

Santos da Silva, P.; Pedreira, I.; de Brito, L.; Soares J. (2011). Incubação de operadora de receptivos populares especializada em roteiros turísticos urbanos alternativos no bairro do Cabula e entorno, em Salvador-Bahía. XI Congresso Luso Afro Brasileiro de Ciencias Sociais. Universidade Federal da Bahía.

Sampaio, C. A. (2005). Turismo como fenômeno humano: princípios para se pensar a socioeconomia sob a denominação turismo comunitário. Santa Cruz do Sul (RS): EDUNISC, v. $1.146 \mathrm{p}$

Skewes, J. C.; Henríquez, C., y Pilquimán, M. (2012). Turismo comunitario o de base comunitaria: Una experiencia alternativa de hospitalidad vivida en el mundo mapuche. Tralcao sur de Chile. Revista de Cultura y Turismo Año $6 \mathrm{~N}^{\circ}$. 2: 73-85. 